\title{
The W7-X Stellarator Project
}

\author{
F. Wagner \\ Max-Planck-Institut für Plasmaphysik, Garching \\ EURATOM Association
}

Design work in preparation for Europe's next major step in stellarators as an alternative for magnetically confined plasma fusion has demonstrated that advanced devices can be optimized.

Europe is following two strategic paths on its way to demonstrating a commercially viable fusion reactor for electric power generation. One path envisages energy production at the GW level in the shortest possible timescale using a self-sustaining, energy producing plasma (a so-called burning plasma). To achieve this important goal, the four major fusion programmes (Europe, USA, Japan and Russia) have merged their knowledge and will jointly build and operate the International Thermonuclear Experimental Reactor (ITER). The device will demonstrate the feasibility of large-scale fusion energy production and will address questions related to the physics and fusion technology of the burning plasma state, notably ignition and control, plasma stability in the presence of a large population of highenergy particles, $\alpha$-particle losses, power exhaust, and tritium breeding. ITER will be a tokamak since this configuration is at the most advanced stage of development and provides the most credible basis for extrapolating to the size and requirements of a burning plasma.

Although the ITER line has the best prospects for demonstrating and exploring a burning plasma it nevertheless admits to drawbacks with respect to an economically viable fusion reactor. Being founded on existing data, ITER represents the highcurrent tokamak line which is unlikely to lead to a reactor operating in the steadystate. Moreover, its plasma is easily disrupted (i.e., prone to sudden current quenches) and performance peaks in the high-current corner of operational space. So ITER will be forced to walk along a brink: any loss of equilibrium will incur a current quench within a few milliseconds accompanied by mechanical and electrical

Friedrich Wagner is responsible for the experimental aspects of work on the W7-AS stellarator at the MaxPlanck-Institut für Plasmaphysik (IPP), BoltzmannStrasse 2, D-85748 Garching. He is a member of the IPP Directorate and has been an Honorary Professor at the TU Munich since 1993. Professor Wagner graduated in 1970 from TU Munich where he was awarded a Ph.D. in low-temperature physics in 1972. He joined the IPP in 1975 after spending two years at Ohio State University, USA, becoming the project head of ASDEX in 1986 and a Fellow of the MaxPlanck Gesellschaft in 1988. hazards. The device will be designed to withstand disruptions, but their frequency has to be limited.

\section{Concept Improvement Tokamaks}

The deficiencies of the ITER line - difficulty of steady-state operation, the stresses accompanying disruption of the plasma, and the large size of a high-current device - are being tackled in Europe's second path, which is called concept improvement. Existing concepts satisfy the physics requirements and provide some of the technology for the most urgent next step, namely realization of a burning plasma. But improvements are needed and in the case of tokamaks, they should lead to the so-called Advanced Tokamak offering superior confinement and stability in smaller, low-current devices. Rigourous optimization of the mag- netic and kinetic ingredients in turbulent transport and of magneto-hydrodynamic stability is essential. Unfortunately, many critical aspects can only be assessed using empirical data.

A self-sustaining bootstrap current is of utmost importance in tokamaks. Such devices are based on a strong toroidal current flowing within the plasma which provides a poloidal field component superimposed on the toroidal one to establish a rotational transform, where magnetic field lines twist and wind helically to embrace the torus. The current is called a bootstrap current because it flows without external excitation, being generated by pressure differences within the plasma and not, as is usually the case, by induction as in a transformer. The strategy for optimizing advanced tokamaks is centred on a large bootstrap current. If the fraction of the total current provided by the bootstrap current is large it might be possible to design a steady-state, low-current tokamak that requires a moderate external current drive (such a current is created by injecting either particles or high-frequency waves).

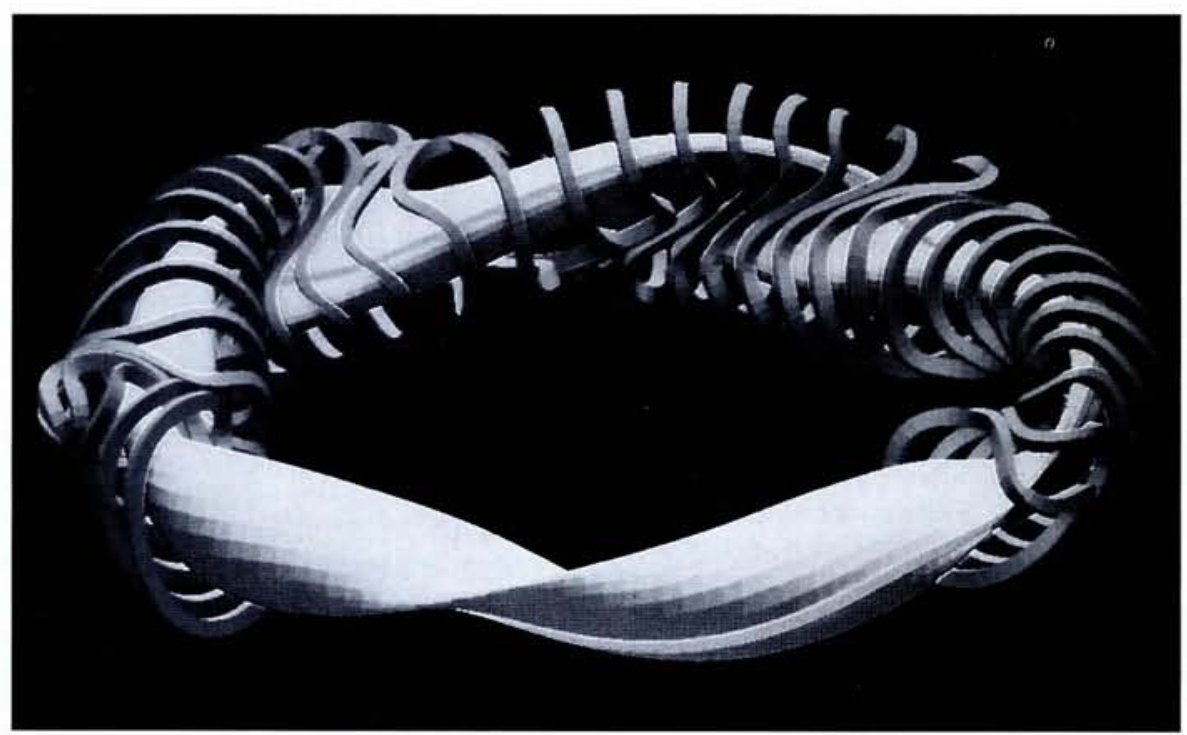

Fig. 1 - The outermost flux surface of the W7-X stellarator with a band of field lines and some of the modular coils. The W7-X's five toroidal field periods will maintain a plasma with obvious poloidal field components and a strong indentation such that the plasma is beanshaped at the corners of the pentagon. The rotational transform of W7-X is chosen to be about 1 as this value allows the safe realization of maximal magnetic shear. The major radius of the device will be $5.5 \mathrm{~m}$ at an aspect ratio of 10. As stellarators are made for steady-state operation, W7-X will have superconducting coils. The maximum field is $3 T$ and is matched to the present electron cyclotron heating frequency of $140 \mathrm{GHz}$. 


\section{Stellarators}

Stellarators represent Europe's second approach for confining toroidal plasmas. They aim to do this using magnetic fields generated by currents lying outside the plasma region. They share with the tokamak the basic concept of nested magnetic surfaces for achieving confinement, but a net toroidal plasma current is not required. Such devices are especially attractive since they provide intrinsic steady-state operation and are inherently disruption-free. However, the classical stellarator based on Lyman Spitzer's original design published in 1958 also requires concept improvement to qualify as a commercially viable reactor.

The basic element of the stellarator coil system is a helical coil which embraces the toroidal plasma. The suitability of helical systems for reactors has been questioned from the technical point of view since they are difficult to fabricate and are operated close to the technical limits: a modular design based on a set of single coils is therefore essential. The modular coils inevitably have to be non-planar because they also provide the poloidal field component. Such an approach would facilitate optimization, thereby removing one of the major drawbacks of the classical stellarator.

Stellarators (cover illustration) form part of the concept improvement programme and several major issues are being tackled. The plasma cross-section in a classical stellarator has a larger aspect ratio than for tokamaks, and the twist angle of rotational transform in the plasma core is generally smaller. As a result, the equilibrium of the nested flux surfaces suffers from a large outward shift of the core region (the Shafranov shift) and operation is limited to low values of the ratio $\beta$ of the plasma to magnetic field energy. Indeed, stellarators have demonstrated $\beta$-values in the range of $2 \%$, whereas tokamaks have been operated beyond $12 \%$ (economically viable reactors of both configurations are assumed to have $\beta$-values of around $5 \%$ ).

A critical problem for classical stellarators is collisional transport in the outward direction arising from binary Coulomb collisions in the long mean-free path conditions of a reactor plasma. Particle and energy transport are governed by the drift of particles trapped in the local helical field component, which leads to fluxes much larger than those arising in tokamaks. Although the level of ion transport is reduced by the ambipolar radial electric field, it still remains too high under reactor conditions.

Unacceptably large collisionless $\alpha$-particle losses are another characteristic consequence of a stellarator's field configuration. In an axisymmetric tokamak, all particle orbits are confined as long as collisions do not play a role. This is not the case for a truly three-dimensional stellarator where essentially every reflected particle is lost without collision. Reflection of the particles is caused by the field gradient along the field lines, as in a mirror plasma device where reflection is in fact the basis for confinement.

\section{The Wendelstein Devices}

The first German experiments on stellarators began in 1961 at the Max-Planck Institute for Physics and Astrophysics in Munich, and have continued since 1965 at the Institute for Plasma Physics, Garching. The Wendelstein 7-X device (cover illustration and Fig. 1), presently in the engineering design phase, is being planned as the world's largest stellarator experiment. A similarly sized Large Helical Device (LHD) is under construction at a green-field site in Japan and the small, very flexible TJ-II stellarator is nearing completion in Madrid.
W7-AS

Some of the design elements of W7-X have already been tested in its predecessor, W7-AS. This device has demonstrated that equilibrium can be improved by reducing the equilibrium current $j_{\|}$that flows parallel as opposed to perpendicular to the field lines (the ratio $\left\langle j_{\|}{ }^{2}\right\rangle \mid\left\langle j_{\perp}^{2}\right\rangle$, which is typically 10 for a classical stellarator, has been decreased to $\approx 3$ in W7-AS and will be further reduced to $\approx 0.5$ in W7-X). W7-AS has been operated at high $\beta(<\beta>\leq 1.4 \%)$, beyond the operating regime for a comparable classical stellarator and without meeting a limit. It has verified the technical feasibility of modular coils and paved the road toward tackling issues which unlike the classical physics issues cannot be subjected to an optimization scheme.

\section{Optimizing Stellarators}

Stellarators need optimizing because classical physics issues and technical problems limit their viability as fusion devices. Recent work at the Institute for Plasma Physics, Garching, has shown that optimization is possible based on fundamental concepts developed within the IPP group working on the theory of stellarators. Optimization requires the solution of boundary value problems where the geometry of the outermost closed magnetic surface determines the properties of the enclosed confinement volume as well as the shapes of the modular coils (engineering constraints enter at this point). Optimization of the proposed Wendelstein W7-X stellarator and the inherent physics have been reported in detail in [1] while [2] introduces the equilibrium and stability aspects of toroidal systems.

Optimization aims to satisfy a number of physical requirements including:

- Smoothly nested flux surfaces free of low- and medium-order resonances. Within this requirement, the magnetic shear should be maximal to avoid undue sensitivity to field perturbations.

- A low Shafranov shift to allow operation at high $\beta$ while retaining good equilibrium conditions. The goal is high stability for $\beta$-values of up to $5 \%$.

- Collisional transport, particularly in the long mean-free path regime, has to be reduced below the possible anomalous level.

- A small bootstrap current so that the major design properties of the magnetic configuration are not lost on moving from low to high $\beta$.

- Assured confinement of $3.5 \mathrm{MeV} \alpha$-particles in a future reactor during their thermalization time of about $0.1 \mathrm{~s}$.

The optimization process yields details of the plasma shape such as its aspect ratio, number of periods, variation of the cross-section along the toroidal direction (field mirror ratio) and the form of the flux surfaces (superimposition of different helical components which lead to helical excursion, ellipticity, triangularity, and indentation). As neo-classical transport effects are determined by the variation of magnetic field $|B|$ within a flux surface, the magnetic "rigidity" provided by the reduction of $j_{\|}$ensures low levels of the related fluxes for low and high $\beta$. Other considerations include:

- A small Shafranov shift requires a reduction of the equilibrium current $j_{\|}$which flows along the field lines and gives rise to a perturbing vertical field. It is achieved by adjusting the curvature of the helical field and the ellipticity of the cross-section.

- Stability at high $\beta$ relies on stabilization by the magnetic well provided by the helical curvature combined with plasma shaping. Reduction of $j_{\|}$also favours stability at high $\beta$. - Symmetry (axisymmetry or helical symmetry), decisive for confining trapped particles, is however not the only means for achieving acceptable $\alpha$-particle confinement and a low level of transport at long mean-free paths. Reduction of radial drifts away from the flux surface can also be achieved in truly three-dimensional configurations by proper combinations of the toroidal and helical curvatures and by localizing trapped particles in the region of low toroidal field variation. The ripple of about $10 \%$ in the superimposed magnetic field which causes the radial drifts is reduced to an effective ripple of $<2 \%$.

- The bootstrap current, another neo-classical effect, can be kept low in a similar way. Toroidal and helical curvature alone give rise to undesirably large bootstrap currents, but of opposite sign. Combination of toroidal and helical curvature basically allows elimination of the bootstrap current.

[1] Nührenberg J. et al., Plasma Physics \& Controlled Fusion (to be published); Grieger G. et al., Physics of Fluids B 4 (1992) 2081; Wobig H., Plasma Physics \& Controlled Fusion 35 (1993) 903. [2] Gruber R., Europhys. News 22 (1991) 93. 
W7-AS has been successfully operated in the high-confinement mode ( $\mathrm{H}$-mode), a region of improved thermal insulation [Wagner F., Europhys. News 17 (1986) 48] which is the most relevant tokamak confinement regime. This is the first time that the $\mathrm{H}$-mode has been achieved outside the tokamak family. The W7-AS device at Garching has also demonstrated ultra-high density operation and the possibility of utilizing the natural islands residing at the edge of the plasma to divert the fluxes crossing the last closed flux surface.

\section{$W 7-X$}

The W7-X stellarator is an experimental device (Fig. 1). It will not only deliver many new results important to successful continuation of the stellarator line but has also to demonstrate that its optimization based on theoretical concepts is indeed feasible (optimization - see insert - became possible with the introduction of modern computers). Work carried out so far has demonstrated that the various goals of the optimization process, which occasionally conflict, can be met in a single device. Experimental flexibility is incorporated so that stability aspects of the optimized magnetic architecture such as shear (spatial variations of the field-line pitch) and magnetic well (the minimum of magnetic field at the plasma centre) can be changed. Exhausting of plasma impurities can be studied and modified by operating with a series of edge islands or with a more ergodic edge configuration for the so-called divertor used to define the structure of the magnetic field surfaces at the edge of the plasma. Moreover, resonating field lines can be placed in the core of the plasma to simulate, if necessary, the effect of sawteeth-like variations of the core parameters on impurities, or at its edge to simulate a divertor configuration comprising a single island.

It will also be interesting to explore the effect of optimized drift orbits on the mechanisms leading to turbulent transport. The characteristics of trapped particles in turbulent transport can be changed by operating at various ratios of the minimum to the maximum field strengths (mirror ratio). It is important to note that the resonance of trapped particles is avoided in W7-X because diamagnetic fluid flow and trapped particle poloidal precession are of opposite sign.

The availability of $10 \mathrm{MW}$ of steady-state electron cyclotron resonance heating $(E C R H)$ will allow the study of plasmas under reactor-relevant, long mean-free path conditions, and $20 \mathrm{MW}$ pulsed neutral beam injection will permit a systematic investigation of MHD stability and the $\beta$-limit. The $\mathrm{ECRH}$ current drive can be used to provide a toroidal current of 50-100 kA, which will add further experimental flexibility for investigating MHD and confinement issues while allowing stellarator physics to be linked to the tokamak world.

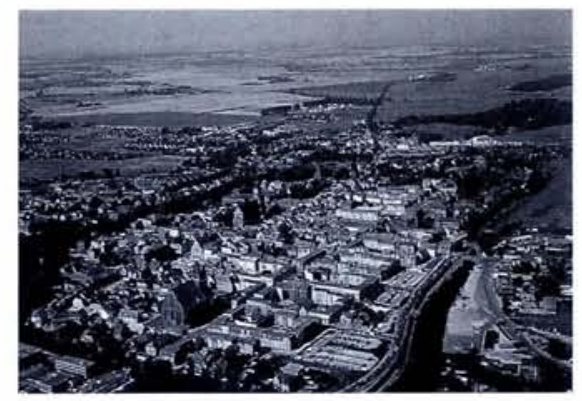

Fig. 2 - Bird's eye view of the city of Greifswald on the Baltic Sea. Greifswald is a university town where plasma physics is well established in the physics faculty of Ernst Moritz Arndt University. The Institute of Low-Temperature Plasma Physics of the former east German Academy of Sciences is also located in Greifswald, and the site of a disused nuclear plant at Lubmin some 20 kilometres from Greifswald has been proposed for the ITER reactor.

\section{Status of the W7-X Project}

W7-X was approved by the IPP in 1990 and received restricted Phase I approval by EURATOM review panels in 1991. One crucial question remained unanswered at that time, namely how W7-X would fit into the European fusion strategy. It was answered in the frame of a detailed assessment of the European fusion programme for 1994-1998, where W7-X is proposed as the major new project to be started in this period. The project has meanwhile received full Phase I approval and the Phase II process, the final step in the European assessment procedure, has begun. Members of the European Associations have been invited to join the IPP in the pioneering enterprise to realise the $W 7-X$

If this final step is passed successfully the first plasma can be expected in 2002. W7-X will, however, not be built at the IPP, Garching, the site of present German stellarator research where the optimization idea was born. It will most likely be located in Greifswald (Fig. 2) following another optimization process which has to do with the research structure in Germany after reunification. The national financial support (55\%) is being provided under the condition that W7-X is treated by the German fusion community as a contribution to the development of research structures in the eastern part of Germany.

The IPP has been charged with the task of founding an extension at Greifswald and operating the institute within the framework of the Max-Planck Gesellschaft, which has already given its approval. As "W" stands for Wendelstein, a prominent mountain in the Bavarian Alps, the device would then become a symbol that fusion is indeed capable of moving mountains.

\section{ANOMALOUS TRANSPORT}

\section{European Task Force Formed}

The ubiquitous anomalous transport processes observed in magnetically confined plasmas have defied a convincing explanation. The US Transport Task Force (TTF) inaugurated in 1989 has been a very successful in harnessing US resources to deve lop understanding. Working groups comprising experimentalists, diagnostic experts, theoreticians, and modellers from participating institutes concentrate on specific topics, such as the physics of the transition from the low-confinement mode (L-mode) to the highconfinement mode $(\mathrm{H}$-mode). The groups then report at the TTF annual meeting. This focussed and collaborative mode of working has already clarified many facts, although many questions remain unanswered - indeed new ones have arisen!

There is also considerable activity within the European Fusion programme addressing transport issues. The question of enhanced co-operation between the various European groups involved has naturally arisen; formal links between Europe and the US are another possibility.

A discussion, led by Friedrich Wagner (Institute for Plasma Physics - IPP, Garching) and attended by Ben Carreras, head of the US TTF, at a workshop organized by Jan Weiland in Göteborg last June considered the possibility of a European TTF. While there was general support for the idea it was recognized that the situation in Europe was very different: a simple replica of the US organization was inappropriate. Nevertheless, stronger collaboration between existing programmes would enhance European work. A number of topics, mainly those being treated by existing collaborations, were suggested and individuals offered to take the lead in building on these collaborations and focusing their efforts to clarify important issues. The readiness of the theorists to join these "topical groups" was particularly encouraging. Interactions with the US TTF would involve information exchange rather than active participation in joint working groups.

Additional meetings and costs would be avoided and existing conferences and workshops should be used to develop collaborations within the topical groups and with members of the US TTF. However, it was envisaged that significant progress would be reported at successors to the Göteborg meeting, namely the annual meetings of the US TTF and a new, biannual joint meeting in Europe (the first will be held in 1996, possibly in Vienna). The IPP Jülich, IPP Garching and Chalmers University have volunteered to 